\title{
White Matter Fiber Degradation Attenuates Hemispheric Asymmetry When Integrating Visuomotor Information
}

\author{
Tilman Schulte, ${ }^{1}$ Eva M. Müller-Oehring, ${ }^{1,2}$ Torsten Rohlfing, ${ }^{1}$ Adolf Pfefferbaum, ${ }^{1,2}$ and Edith V. Sullivan ${ }^{2}$ \\ ${ }^{1}$ Neuroscience Program, SRI International, Menlo Park, California 94025, and ${ }^{2}$ Department of Psychiatry and Behavioral Sciences, Stanford University \\ School of Medicine, Stanford, California 94305
}

\begin{abstract}
Degradation of white matter fibers can affect the transmission of signals in brain circuits that normally enable integration of highly lateralized visual and motor processes. Here, we used diffusion tensor imaging tractography in combination with functional magnetic resonance imaging to examine the specific contributions of interhemispheric and intrahemispheric white matter fibers to functional measures of hemispheric transfer and parallel information processing using bilateral and unilateral left and right visual field stimulation in normal and compromised systems. In healthy adults, a greater degree of bilateral processing advantage with the left (nondominant) hand correlated with higher integrity of callosal fibers connecting occipital cortices, whereas less unilateral processing advantage with the right hand correlated with higher integrity of left-hemispheric posterior cingulate fibers. In contrast, alcoholics who have compromised callosal integrity showed less bilateral processing advantage than controls when responding with the left hand and greater unilateral processing advantage when responding with the right hand. We also found degraded left posterior cingulate and posterior callosal fibers in chronic alcoholics, which is consistent with functional imaging results of less left posterior cingulate and extrastriate cortex activation in alcoholics than controls when processing bilateral compared with unilateral visual field stimulation. Together, our results demonstrated that interhemispheric and intrahemispheric white matter fiber pathways mediate visuomotor integration asymmetrically and that subtle white matter fiber degradation in alcoholism attenuated the normal pattern of hemispheric asymmetry, which may have ramifications for the efficiency of visual information processing and fast response execution.
\end{abstract}

\section{Introduction}

To explore our visual environment, the brain integrates information from each visual hemifield via the contralateral cerebral hemisphere (Wandell et al., 2007). Although basic visual stimuli are processed bilaterally (de Lacoste et al., 1985; Corballis et al., 2002), asymmetrical processing arises for high-level visual representations to optimize processing quality, speed, and capacity (Mevorach et al., 2005). The degree to which activity between the hemispheres is symmetrical or asymmetrical may depend on the integrity of corpus callosum fibers connecting the hemispheres (Stark et al., 2008).

Splenial fibers have extensive projections to posterior cortical regions, including extrastriate cortex (Pandya and Seltzer, 1986; Hofer and Frahm, 2006; Park et al., 2008), and may subserve parallel processing and integration of visual information presented separately to each hemisphere (Schulte et al., 2005) (Fig. 1). Asymmetric callosal projections from primary visual (Putnam et al., 2010) and motor (Guye et al., 2003) cortices may provide

Received April 26, 2010; revised July 23, 2010; accepted July 24, 2010.

This work was supported by National Institute on Alcohol Abuse and Alcoholism Grants AA018022, AA010723, AA005965, AA012388, and AA017168 and National Institute of Biomedical Imaging and Bioengineering Grant EB008381. We thank Margaret Rosenbloom for critical reading of and valuable comments on this manuscript. We also thank Shara Vinco for excellent technical experimental assistance and Dr. Stephanie Sassoon for clinical screening of study participants.

Correspondence should be addressed to Dr. Tilman Schulte, SRI International, Neuroscience Program, 333 Ravenswood Avenue, Menlo Park, CA 94025-3493. E-mail: tilman.schulte@sri.com.

DOI:10.1523/JNEUROSCI.2160-10.2010

Copyright $\odot 2010$ the authors $\quad 0270-6474 / 10 / 3012168-11 \$ 15.00 / 0$ functional asymmetries, such as faster right-to-left than left-toright interhemispheric transfer time for visuomotor information (Barnett and Corballis, 2005) or a left-hemispheric superiority for handedness (Tommasi, 2009). Integration of lateralized visual input and motor output may be further mediated by intrahemispheric connectivity involving fibers connecting the visual area of medial parieto-occipital cortices (Galletti et al., 2003; Gamberini et al., 2009) and supplementary motor area (Haaxma and Kuypers, 1975). Whether individual variation in interhemispheric and intrahemispheric white matter fibers, determined with in vivo fiber tracking, mediates functional brain asymmetry and visuomotor integration has yet to be tested.

The anatomical arrangement of the human visual system permits examination of independent contributions from each hemisphere for the integration of parallel visuomotor processes and can be assessed by comparing paired with single targets presented to one or both visual hemifields (Miller, 1986; Mordkoff and Yantis, 1991). In a previous functional magnetic resonance imaging (fMRI) study, processing advantages occurred for paired stimuli simultaneously presented in both hemifields relative to the sum of the two stimuli presented singly in each hemifield and were associated with enhanced activation of bilateral extrastriate cortices, suggesting interhemispheric neural summation (Schulte et al., 2006). Because efficient transmission of signals in circuits for sensory and motor processing depends on the integrity of cerebral fiber tracts (Barrick et al., 2007; Turken et al., 2008), subtle disruption of callosal fiber integrity, such as seen in alcoholism (Wang et al., 2009; Pfefferbaum et al., 2010), can disturb the 


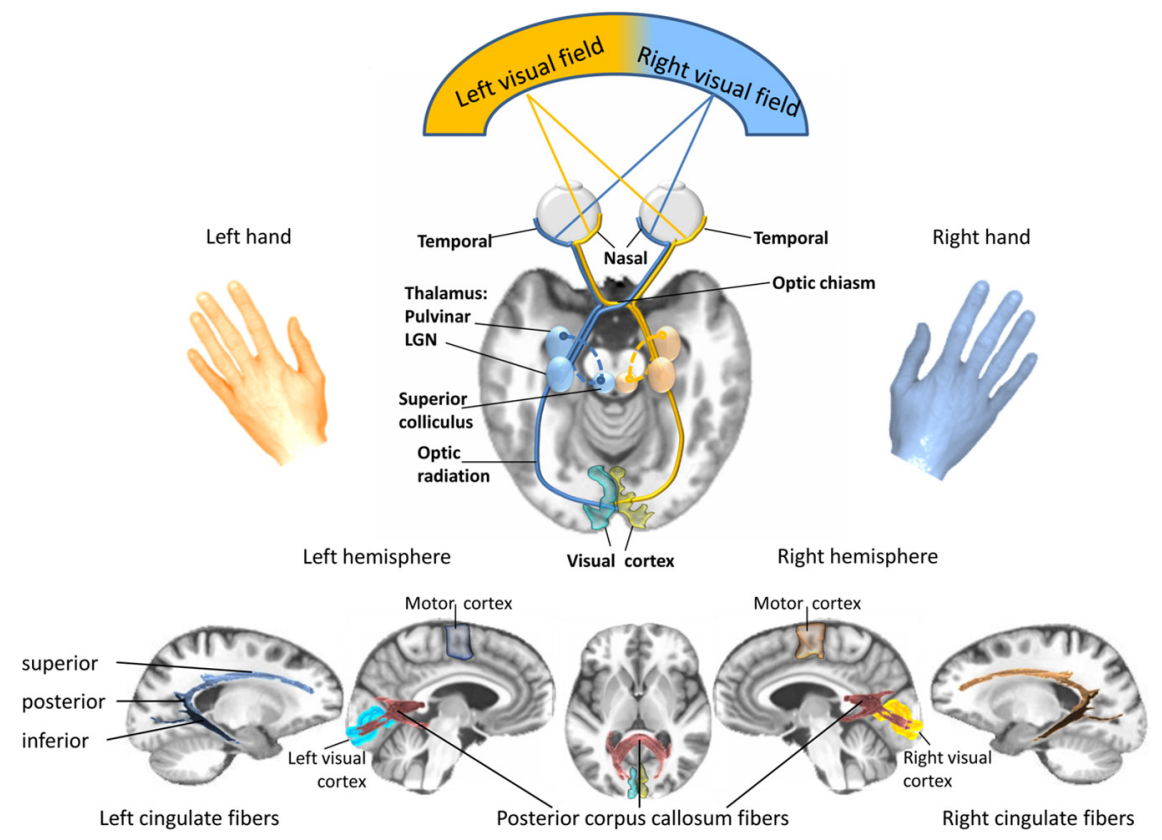

Figure 1. Top, Schematic representation of the highly lateralized visual projection system. The visual information from each visual hemifield is projected to the visual cortex of the contralateral hemisphere; it is transmitted from the retina along the optic nerve through the optic chiasm, where fibers from the nasal retinal nerve cells cross to join the fibers from the temporal retinal nerve cells of the other eye. This way, the brain merges visual information from one visual hemifield to form an optic tract, which travels from the chiasm to the lateral geniculate nucleus (LGN) in the thalamus and then along the optic radiation fibers to the visual cortex of each cerebral hemisphere. Bottom, Illustration of posterior corpus callosum fibers, and cingulate fiber bundles for interhemispheric and intrahemispheric integration of visuomotor information within dynamically co-operating brain networks. Our results indicate that at the cortical level, between-hemisphere visuomotor integration is mediated cooperatively via posterior corpus callosum fibers and within-hemisphere integration is conveyed via left posterior cingulate fibers in an inhibitory fashion. Individual variation of white matter fiber structure modulates visuomotor integration dynamically and in interaction with a dominant left-hemispheric motor output system. Subtle white matter fiber degradation, as seen in alcoholism, contributes to an attenuation of the normal functional brain asymmetry.

normal balance of interhemispheric and lateralized cognitive processes (Schulte et al., 2008).

To examine the contribution of interhemispheric and intrahemispheric fiber tracts to functional measures of hemispheric transfer and integration, we used diffusion tensor imaging (DTI)based tractography and fMRI. Recovering chronic alcoholics are known to have disrupted white matter fiber tract integrity without frank lesions (Sullivan and Pfefferbaum, 2005) and provide a model population in which to test hypotheses about interhemispheric and intrahemispheric information processing. We hypothesized that disruption of white matter fiber tracts related to alcoholism would impair bilateral and enhance unilateral processing advantage, thereby impeding interhemispheric integration and the normal pattern of functional asymmetry, verified with functional imaging.

\section{Materials and Methods}

\section{Study participants}

Groups comprised 17 alcoholic men and 16 control men. Alcoholics were recruited from local rehabilitation programs, and controls were volunteers from the local community. All subjects were screened with the Structural Clinical Interview for Diagnostic and Statistical Manual of Mental Disorders, fourth revision (SCID) (American Psychiatric Association, 2000) and a clinical examination to rule out other Axis I diagnoses or nonalcohol substance abuse. Subject groups were matched in age, handedness, and other demographic variables (Table 1). All subjects were right handed except for two (one control and one alcoholic), who self-reported right handedness but scored in the non-right-handed range as determined from an objective quantitative questionnaire (Crovitz and
Zener, 1962). The National Adult Reading Test, an estimate of premorbid intelligence, did not distinguish controls and alcoholics (Table 1), who were neither demented nor amnesic. Lifetime alcohol consumption was estimated using a semistructured interview and was expressed in kilograms (Pfefferbaum et al., 1988). Drinks of each type of alcoholic beverage were standardized to units containing $\sim 13.6 \mathrm{~g}$ of alcohol and summed over the lifetime. Two controls without quantitative drinking histories underwent a clinical interview with the SCID, which inquired about alcohol and other drug use. Subjects gave written informed consent to participate in this study, which was approved by the institutional review boards at SRI International and Stanford University School of Medicine.

Stimuli and experimental design

Study participants underwent visual testing and all structural and functional imaging protocols in a clinical whole-body 3T scanner (General Electric). Using a back-projection system, participants viewed stimuli on a mirror attached to the head coil. They were instructed to fixate on a point in the middle of the display throughout the test and to have feet, legs, and arms separated to minimize the possibility of cross-cueing. The task was to press a button (Button-Pad; PsyScope) as fast as possible whenever a stimulus appeared, whether a single or a paired event. The interstimulus interval varied among 500, 700, and $900 \mathrm{~ms}$. All stimuli were displayed for $150 \mathrm{~ms}$. Stimuli were filled white disks on a dark gray background localized left and right of the fixation point and above and below the horizontal meridian (to avoid the blind spot) and were presented singly or in pairs to the left and right visual hemifield (bilateral stimulation) and to the same hemifield ipsilateral to the responding hand (unilateral stimulation). Two runs were presented, one requiring a right-hand response and the other a left-hand response; the run order (i.e., "right hand-left hand" vs "left hand-right hand") was counterbalanced across subjects. Each run comprised 20 blocks of nine trials (eight stimuli, one fixation trial) for each condition block: single left, single right, paired bilateral, single unilateral, paired unilateral (Fig. $2 a$ ).

The task comprised 360 trials in total: 160 stimulus trials and 20 fixation trials for each run. Each run contained 32 single left, 32 single right, 32 paired bilateral, 32 single unilateral, and 32 paired unilateral stimulus presentations. Stimulus blocks were intermixed. Fixation performance was assessed using a brief color change of the fixation point in the middle of the screen between trials (fixation trial). Reaction times (RTs) $<100$ $\mathrm{ms}$ (anticipations) and $>3 \mathrm{SD}$ (prolonged responses) were excluded from analysis.

Crossed-uncrossed difference and redundant targets effect. Behavioral testing elicited the crossed-uncrossed difference (CUD), a behavioral index of interhemispheric transfer time (IHTT) (Poffenberger, 1912), and the redundant targets effect (RTE), a behavioral index of visuomotor integration, which describes faster responses to paired than single stimuli, and was tested with bilateral and unilateral visual field stimulation. The CUD provides a behavioral index of IHTT based on the notion that the uncrossed response (i.e., left hemifield/left hand, right hemifield/ right hand) can be processed within the same hemisphere, whereas crossed responses (i.e., left hemifield/right hand, right hemifield/left hand) require transfer of visuomotor information between hemispheres. Subtracting the sum of crossed responses from the sum of uncrossed responses and dividing this difference by 2 computed the CUD. Subtracting responses to paired stimulation from the mean of the two 
Table 1. Means and SDs of demographic data of the two study groups: chronic alcoholics (ALC) and controls (CTL) ${ }^{a}$

\begin{tabular}{|c|c|c|c|c|c|c|}
\hline Group & Age & Education & NART IQ & Handedness CROVITZ & Visual acuity & Lifetime alcohol consumption (kg) \\
\hline ALC & $51(7.2)$ & $15(2.9)$ & $111(7.8)$ & $21(5.2)$ & $1.3(0.7)$ & $1538(600.2)$ \\
\hline CTL & $49(15.3)$ & $16(2.3)$ & $116(6.6)$ & $21(9.3)$ & $1.8(0.9)$ & $22(35.0)$ \\
\hline p & n.s. & n.s. & n.s. & n.s. & n.s. & 0.0001 \\
\hline
\end{tabular}

${ }^{a}$ Between-group $t$ test: significance at $p<0.05$, two-tailed n.s; not significant.

${ }^{b}$ CROVITZ score: 14-32 right-handed, 50-70 left handed (Crovitz and Zener, 1962).

\section{a Design}

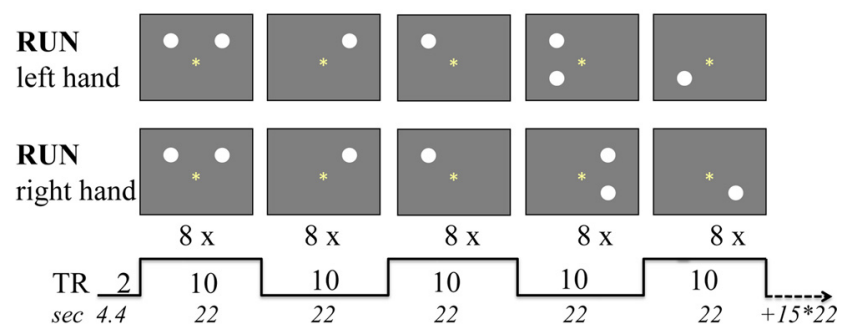

b Contrasts

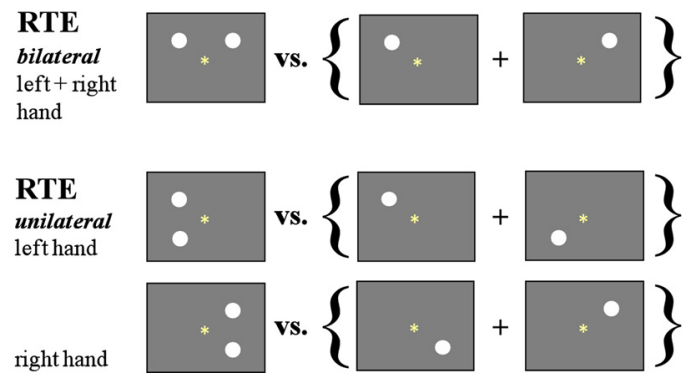

Figure 2. $\quad \boldsymbol{a}$, Redundant targets paradigm: stimulus parameter timing. $\boldsymbol{b}$, Statistical Parametric Mapping contrasts for interhemispheric and intrahemispheric integration.

single stimulations calculated the RTE. RT differences in RTE reflect the individual's overall response facilitation from redundant targets in milliseconds.

Enhanced RTE. We further analyzed the RTE according to the horse race measure $R(t)$ (Miller, 1982). The race model assumes two stochastically independent processes; RT is initiated when information processing of either one of two stimuli is completed. If the shorter response to paired targets depends on the winner of a race between two independent processes initiated by either of two stimuli ( $\mathrm{S} 1$ or $\mathrm{S} 2$ ), then the race model predicts that the cumulative probability of observing a response to redundant targets (S1 and S2) by time $t$ would be less than or equal to the sum of the cumulative probabilities for detecting each component by time $t$ :

$$
P\left(\mathrm{RT}_{\mathrm{S} 1 \text { and } \mathrm{S} 2} \leq t\right) \leq P\left(\mathrm{RT}_{\mathrm{S} 1} \leq t\right)+P\left(\mathrm{RT}_{\mathrm{S} 2} \leq t\right) .
$$

Equation 1 provides an upper limit of the gain achieved by the detection of redundant targets. By transforming Equation 1 into Equation 2, we can test whether the shortening of RTs under double stimulation conditions violates the predictions of the race model by comparing the cumulative distribution function for redundant $\operatorname{targets} P\left(\mathrm{RT}_{\mathrm{S} 1 \mathrm{~S} 2}\right)$ with the sum of cumulative distribution functions for single targets, $P\left(\mathrm{RT}_{\mathrm{S} 1}\right)+$ $P\left(\mathrm{RT}_{\mathrm{S} 2}\right)$ :

$$
R(t)=P\left(\mathrm{RT}_{\mathrm{S} 1 S 2}\right)-\left[P\left(\mathrm{RT}_{\mathrm{S} 1}\right)+P\left(\mathrm{RT}_{\mathrm{S} 2}\right)\right] .
$$

In Equation 2, the race measure $R(t)$ can only be evaluated when $P\left(\mathrm{RT}_{\mathrm{S1}}\right)+$ $P\left(\mathrm{RT}_{\mathrm{S} 2}\right)<1$. Thus, the range of possible values of $R(t)$ is -1.0 to 1.0 . To evaluate whether the shortening of RTs with redundant targets violates the predictions of the race model, we used this race measure, $R(t)$. The graph of $R(t)$ was calculated for each subject by ranking the RTs for the three stimulus conditions (S1, S2, and S1S2). We defined a bin consisting of 8 RTs and computed a cumulative distribution function, counting the number of stimuli in each condition in the first bin containing the 8 shortest RTs, in the second bin containing the 16 shortest RTs, and so on, cumulatively. Positive $R(t)$ values indicate a higher number of fast responses elicited by bilateral than by unilateral trials within one bin (i.e., the amount of violation). To quantify violation, we used the amplitude of the violation graph $R(t)$ [peak value of $R(t)$ ] (Schulte et al., 2005). The $R(t)$ amplitude reflects the individual's maximal response facilitation.

\section{DTI}

DTI and MRI acquisition. All imaging data were acquired with an eightchannel head coil at $3 \mathrm{~T}$ after higher-order (nonlinear) shimming (Kim et al., 2002). DTI and fast spin echo (FSE) structural data were collected with the same slice locations: DTI, two-dimensional (2D) echo-planar: repetition time (TR), $7300 \mathrm{~ms}$; echo time (TE), $86.6 \mathrm{~ms}$; thickness, $2.5 \mathrm{~mm}$; skip, $0 \mathrm{~mm}$; locations, $62 ; b=0$ [five number of excitations (NEX) $]+15$ noncollinear diffusion directions, $b=860 \mathrm{~s} / \mathrm{mm}^{2}$ (two NEX) +15 opposite polarity noncollinear diffusion directions, $b=860 \mathrm{~s} / \mathrm{mm}^{2}$ (two NEX); FOV, $240 \mathrm{~mm}, \mathrm{x}$-dim $=96, \mathrm{y}$-dim $=96$, reconstructed to $128 \times 128 ; 4030$ total images); FSE, 2D axial: TR, $7850 \mathrm{~ms}$; TE, 17/102 ms; thickness, $2.5 \mathrm{~mm}$; skip, 0 ; locations, 62. The dual-echo FSE sequence acquired as part of the DTI protocol was used for coregistering structural and diffusion images. T1weighted spoiled gradient-recalled acquisition in a steady state (SPGR) [three-dimensional (3D) axial inversion recovery-prep; TR, $6.5 \mathrm{~ms}$; TE, $1.6 \mathrm{~ms}$; thick, $1.25 \mathrm{~mm}$; skip, $0 \mathrm{~mm}$; locations, 124] images were aligned, such that two $1.25 \mathrm{~mm}$ SPGR slices subtended each 2.5-mm-thick FSE/ DTI slice. A field map was generated from a gradient recalled echo sequence pair (TR, $-460 \mathrm{~ms}$; TE, $3 / 5 \mathrm{~ms}$; thickness, $2.5 \mathrm{~mm}$; skip, $0 \mathrm{~mm}$; locations, 62).

DTI analysis. DTI quantification was preceded by eddy current correction on a slice-by-slice basis using within-slice registration, which took advantage of the symmetry of the opposing-polarity acquisition (Bodammer et al., 2004) and also allowed for compensation of the diffusion effect created by the imaging gradients (Neeman et al., 1991), reducing the data to 15 noncollinear diffusion-weighted images per slice for tensor computation. Using the field maps, B0-field inhomogeneityinduced geometric distortion in the eddy current-corrected images was corrected with PRELUDE [Phase Region Expanding Labeler for Unwrapping Discrete Estimates (Jenkinson, 2003)] and FUGUE [FMRIB's Utility for Geometrically Unwarping EPIs (Jenkinson, 2001)].

DTI models water diffusion within each voxel as a zero-mean multivariate Gaussian distribution, which is parameterized by its symmetric $3 \times 3$ covariance matrix, the diffusion tensor. The diffusion-weighted data measured with respect to each of the diffusion gradient directions exhibits a signal attenuation relative to $b=0$ data acquired without diffusion gradients. The logarithm of this signal attenuation is a linear combination of the six unique elements of the diffusion tensor, their weights determined by the direction of the diffusion gradients. Computing a least-squares solution for this overdetermined linear equation system independently at each voxel yielded the elements of the diffusion tensor for that voxel. The diffusion tensor was then diagonalized to obtain eigenvalues $\lambda 1, \lambda 2, \lambda 3$, and corresponding eigenvectors. From the eigenvalues, fractional anisotropy (FA) and two orientational diffusivity measures, $\lambda l$ and $\lambda t$, were calculated on a voxel-by-voxel basis (Pierpaoli and Basser, 1996; Basser and Pierpaoli, 1998; Basser and Jones, 2002). These "native space" DTI data were also used for fiber tracking.

A diffusion tensor can be graphically represented by an equidensity ellipsoid. The axes of this ellipsoid are oriented according to the eigenvectors of the tensor, and their lengths are proportional to the square roots of the corresponding eigenvalues. In tissue that is highly linear, one 
eigenvalue dominates the other two. The ellipsoid is therefore long and narrow and has a preferential orientation, presumed to indicate the course of white matter fiber tracts. FA quantifies the degree to which water diffusion exhibits a predominant orientation: the more linear and organized the fibers in a region, the higher the FA. In an ellipsoid with a preferential orientation, its long axis is referred to as longitudinal, or axial, diffusivity $(\lambda l=\lambda 1)$, which has been shown to be an indicator of axonal integrity. The mean of the short axes of the diffusion ellipsoid is referred to as transverse, or radial, diffusivity $(\lambda t=[\lambda 2+\lambda 3] / 2)$, which is considered a marker of myelin integrity (Song et al., 2002; Sun et al., 2006).

Fiber bundle identification and tracking. Fiber tracking was performed with the software by Gerig et al. (2005) based on the method of Mori and colleagues (Mori and van Zijl, 2002; Xue et al., 1999; Xu et al., 2002). This approach requires the identification of a group of "source" voxels from which streamlines (i.e., fiber tracts) are initiated and propagated throughout the brain following the orientation of the principal eigenvector of the diffusion tensor at each point along the fiber. To describe a particular fiber bundle selectively, a group of "target" voxels is also identified, and only the streamlines that pass through the target are retained. Thus, each identified fiber bundle is required to originate in a source voxel and pass through at least one target voxel.

Fiber tract sources and targets were identified on the FA image of the SRI24 atlas (Rohlfing et al., 2010) (http://nitrc.org/projects/sri24) as follows. The major targets for the commissural tracts were identified as all voxels of the corpus callosum in the midsagittal slice. They were then divided into seven sectors, reflecting divisions determined with histological analysis (Pandya and Seltzer, 1986). Commissural sources were two 3-mm-thick planes, $5 \mathrm{~mm}$ bilateral to commissural targets. The cingulate bundle was identified in three sections: superior, posterior, and inferior. The midpoint voxel of each cingulate section was dilated with a morphological operator to produce a $5 \mathrm{~mm}$ cube as the fiber tracking target. Sources were 3 - $\mathrm{mm}$-thick planes placed $5 \mathrm{~mm}$ anterior and $5 \mathrm{~mm}$ posterior to the targets.

These targets and sources were mapped to the corresponding locations on the native-space DTI images for each subject using coordinate transformations computed by nonrigid image registration (Rohlfing and Maurer, 2003) (http://nitrc.org/projects/cmtk/). In particular, the SPGR channel of the SRI24 atlas was registered to each subject's SPGR image, which was registered to that subject's FSE image pair, which in turn was registered to the $b=0$ image of the subject's DTI acquisition. All three transformations were concatenated to produce sources and targets in the subject's DTI space using only a single reformatting operation.

The tensor data as well as targets and sources were passed to the fiber tracking routine in native space, thus avoiding the need for tensor field resampling and tensor reorientation. Fiber tracking parameters included white matter extraction threshold (minimum FA) of 0.17 , minimum fiber length of $37.5 \mathrm{~mm}$, maximum fiber length of $187.5 \mathrm{~mm}$, fiber tracking threshold of 0.125 (terminates a fiber if the vector field in the local neighborhood is too noisy), and angle of maximum deviation of 0.80 $\left(\sim 37^{\circ}\right.$ maximum angle between successive fiber segments). In postprocessing, we enforced the additional constraint that a commissural fiber originating in a source voxel in one hemisphere was required to pass through a target voxel in the corpus callosum and also through at least one source voxel in the other hemisphere. Similarly, cingulate fibers originating on one side of the cingulate target were also required to pass through at least one source voxel on the other side of the target.

The output of the fiber tracking for each subject and each source-target pair was a 3D geometric model of the fiber paths comprising a table of all point locations along each fiber with local DTI metrics (FA, MD, $\lambda 1, \lambda 2$, $\lambda 3)$. Mean FA, longitudinal diffusivity $(\lambda \mathrm{l})$, and transverse diffusivity $(\lambda t)$ for each fiber bundle were the units of subsequent analysis.

\section{$f M R I$}

fMRI acquisition. Whole-brain fMRI data were acquired with a $\mathrm{T}^{*}$ weighted gradient echoplanar pulse sequence (2D axial; TE, $30 \mathrm{~ms}$; TR, $2200 \mathrm{~ms}$; flip angle, $90^{\circ}$; in-plane resolution, $3.75 \mathrm{~mm}$; thick, $5 \mathrm{~mm}$; skip, $0 \mathrm{~mm}$; locations, 36; FOV, $240 \mathrm{~mm}$; one NEX). The start of the scan was triggered automatically from PsyScope software. Test instructions were reviewed with the subject by the examiner via the scanner's intercom system before the onset of each run. Structural data acquired were a dual-echo FSE (2D axial; TR, 5000 ms; TE, 17/102 ms; thick, 5 mm; skip, $0 \mathrm{~mm}$; $x y$ matrix, 256; flip angle, $90^{\circ}$; locations, 36; FOV, $240 \mathrm{~mm}$; one NEX) used for spatially registering the fMRI data.

fMRI analysis. Image preprocessing and statistical analyses were performed using the SPM8 software package (Wellcome Department of Cognitive Neurology). The fMRI analysis focused on the whole brain. The functional images were subjected to geometric distortion (field map) correction and motion correction. The FSE structural images were coregistered to the mean unwarped and motion-corrected functional image for each subject and segmented into gray and white matter images. Functional and structural gray matter images were normalized to Montreal Neurological Institute space, and volumes were smoothed with a Gaussian kernel of $8 \mathrm{~mm}$ (full-width at half-maximum). Individual statistics were computed using a general linear model approach (Friston et al., 1995) as implemented in SPM8. Statistical preprocessing consisted of high-pass filtering at $44 \mathrm{~s}$, low-pass filtering through convolution with the SPM8 canonical hemodynamic response function, and global scaling. A random-effects analysis was conducted for group averaging and population inference. To this end, one image per contrast was computed for each subject from a design matrix that included estimated individual movement parameters as regressors in addition to stimulation conditions as explanatory variables.

RTE contrasts of interest were "paired versus sum of two single" stimulation and were computed for bilateral-left hand, bilateral-right hand, unilateral-left hand, and unilateral-right hand conditions (Fig. 2b). For second-level group analyses, these RTE contrast images were subjected to a full factorial model involving three factors: group (controls vs alcoholics), RTE stimulation (bilateral vs unilateral), and RTE response hand (left vs right). For within-group analyses, peak-intensity thresholds were set at $P_{\text {uncorr }}=0.001, k \geq 10$; for between-group analyses, thresholds were set at $P_{\text {FWEcorr }}<0.05$ for combined spatial extent and peak intensity (Poline et al., 1997). We used the MarsBar region-of-interest toolbox (http://marsbar.sourceforge.net) to extract the mean parameter estimates from significant clusters to explore the relationship between regional activation and the integrity of closely located white matter fiber bundles.

\section{Statistical analyses}

Group differences in FA and diffusivity measures $(\lambda l$ and $\lambda \mathrm{t})$ were tested with ANOVA. To examine the relationships predicted between DTI measures (FA and diffusivity) and the behavioral measures of interhemispheric (CUD and bilateral RTE) and intrahemispheric transmission (unilateral RTE), we used Pearson correlation in each subject group separately. Relationships between white matter integrity, lifetime alcohol consumption, and CUD were tested one-tailed, based on the assumptions of lower fiber integrity with higher lifetime alcohol consumption, and of faster IHTT with higher fiber integrity. All other relationships were tested two-tailed. The $\alpha$ level was set to 0.05 for all statistical tests, including hypotheses-driven analyses of structure-function relationships. In addition, Bonferroni-corrected $p$ levels were set at $p<0.007$ (two-tailed) or 0.014 (one-tailed) for corpus callosum and $p<0.016$ for intrahemispheric cingulate multiple comparisons.

\section{Results}

\section{Behavioral results}

CUD

The CUD is a behavioral estimate of interhemispheric transfer time, typically indicated by longer RTs to crossed than uncrossed "visual field-response hand" conditions. Although the alcoholics (7.96 $\pm 16 \mathrm{~ms})$ had longer CUDs than controls $(0.42 \pm 19.5 \mathrm{~ms})$, this difference was not significant by ANOVA with group as between-subjects factor and with CUD (crossed, uncrossed) and response hand (left, right) as within-subject factors (Table 2). A significant stimulation-by-response hand interaction indicated longer response times to crossed than uncrossed trials 
Table 2. Crossed and uncrossed reaction time means for left- and right-hand responses for each group: alcoholics (ALC) and controls (CTL) ${ }^{a}$

\begin{tabular}{|c|c|c|c|c|c|c|c|}
\hline \multirow[b]{2}{*}{ CUD } & \multicolumn{3}{|l|}{ CTL } & \multicolumn{3}{|l|}{$\mathrm{ALC}$} & \multirow[b]{2}{*}{ ANOVA } \\
\hline & Left hand & Right hand & CUD & Left hand & Right hand & CUD & \\
\hline Crossed & $385(63)$ & $335(74)$ & & $395(90)$ & $379(120)$ & & $(\mathrm{G})^{b}$ \\
\hline Uncrossed & $363(68)$ & $356(68)$ & & $367(88)$ & 391 (121) & & $F=0.69, p=0.4$ \\
\hline Paired difference & $22.2(31)$ & $-21.4(22)$ & 0.42 & $27.8(23)$ & $-11.9(18)$ & 7.96 & $(2-1)$ \\
\hline$t$ test & $t=2.84, p=0.013$ & $t=-3.93, p=0.001$ & & $t=4.91, p<0.0001$ & $t=-2.69, p=0.016$ & & $F=49.33, p<0.0001$ \\
\hline
\end{tabular}

${ }^{a}$ Values in parentheses are SDs.

${ }^{b} G$, Main effect for group; 2-I, two-way interaction between crossed- uncrossed conditions and response hand.

Table 3. Group means for chronic alcoholics (ALC) and controls (CTL): RTE difference reaction times (single minus paired targets) for RTE in $\mathrm{ms}^{a}$

\begin{tabular}{|c|c|c|c|c|c|}
\hline \multirow[t]{2}{*}{$\overline{\mathrm{RTE}}$} & \multicolumn{2}{|l|}{ Bilateral stimulation } & \multicolumn{2}{|c|}{ Unilateral stimulation } & \multirow[t]{2}{*}{ ANOVA } \\
\hline & Left hand & Right hand & Left hand & Right hand & \\
\hline CTL & $22.6(25.9)$ & $4.0(19.1)$ & $9.5(19.6)$ & $17.3(17.7)$ & $(G)^{b} F=5.16, p=0.03$ \\
\hline ALC & $0.75(19.3)$ & $8.9(20.7)$ & $5.2(21.9)$ & $-0.8(33.5)$ & $(3-$ I) $F=10.69, p=0.003$ \\
\hline$t$ test & $t=2.76, p=0.009$ & $t=0.72, p=0.5$ & $t=0.59, p=0.6$ & $t=1.92, p=0.06$ & \\
\hline
\end{tabular}

${ }^{a}$ Values in parentheses are SDs.

${ }^{b} G$, Main effects for group; 3-I, three-way interaction between group, stimulation, and response hand.

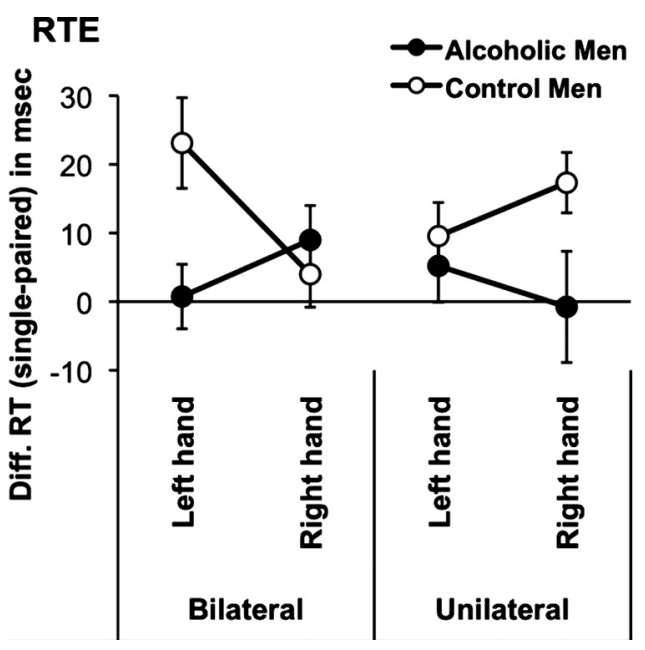

Figure 3. RTEs. Shown is the mean ( \pm SE) for difference (Diff.) RTs $[(S 1+S 2) / 2-S 1 S 2]$ for each condition (bilateral-left hand, bilateral-right hand, unilateral-left hand, unilateral-right hand) and each group (controls, alcoholics).

when responding with the left hand but faster response times to crossed than uncrossed trials when responding with the right hand (Table 2).

\section{RTE}

Given the effect of response hand on CUD, we calculated RTEs for bilateral and unilateral stimulation separately for left- and right-hand responses. For each person, mean RTs to paired stimulation were subtracted from the mean RTs of summed single stimulations. To test whether processing advantages from paired stimulation (RTEs) for these conditions differed between alcoholics and controls, we conducted an ANOVA with group as between-subject factor and hemispheric stimulation (unilateral, bilateral) and response hand (left, right) as within-subject factors. Table 3 and Figure 3 illustrate the results. Processing advantages (i.e., RTEs) were greater for controls than alcoholics. Specifically, for bilateral RTEs, alcoholics showed less response facilitation than controls when responding with the left (nondominant) hand, but not when responding with the right (dominant) hand. The opposite pattern was found for unilateral RTEs: alcoholics showed greater response facilitation than controls when responding with the right hand but not when responding with the
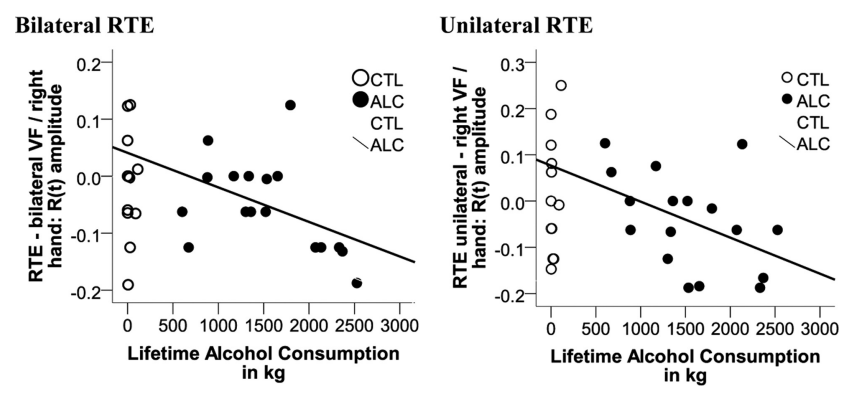

Figure 4. Pearson correlation between lifetime alcohol consumption (kilograms) and the amplitude of violation of the race measure's prediction $R(t)$ by redundant targets in alcoholics (ALC) and controls (CTL).

left hand. This significant three-way interaction was mainly attenuated by the reduced bilateral response facilitation in alcoholics relative to controls when responding with the left hand.

\section{Enhanced RTEs}

We calculated the horse race measure $R(t)$ for each subject (see Eq. 2) to test whether the time of responding to the paired targets was faster than the fastest response to either one of the single targets. $R(t)$ values $>0$ indicate enhanced RTEs that exceed probability and imply a coactivation process. Enhanced RTEs were observed in four to seven controls (23-41\%) and two to six alcoholics (12-35\%) depending on condition (i.e., stimulation and response hand). The number of individuals exhibiting such enhanced RTEs did not differ between groups for any condition $\left(\chi^{2}\right.$ test; all $P>0.2$ ).

\section{Enhanced RTEs and lifetime alcohol consumption}

Pearson correlation tested whether the degree of RTE in alcoholics was associated with the amount of lifetime alcohol consumed. Attenuated RTEs indicated by lower $R(t)$ amplitudes correlated with higher lifetime alcohol consumption ( $\mathrm{RTE}_{\text {unilateral-right hand: }}$ $\left.r=-0.45, p=0.035 ; \mathrm{RTE}_{\text {bilateral-right hand }}: r=-0.45, p=0.034\right)$, a relationship that was not observed in controls $(P>0.17)$ (Fig. 4).

\section{DTI tractography results}

\section{Corpus callosum}

To test for group differences in the integrity of white matter fibers connecting the two cerebral hemispheres, we conducted separate 
A
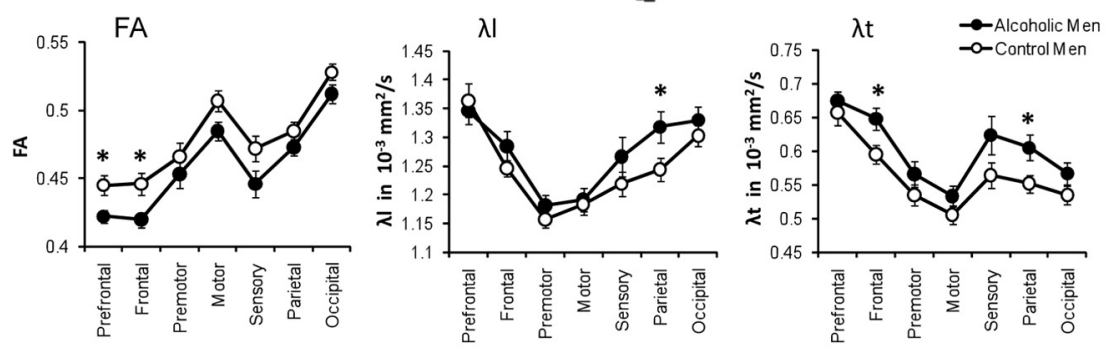

B
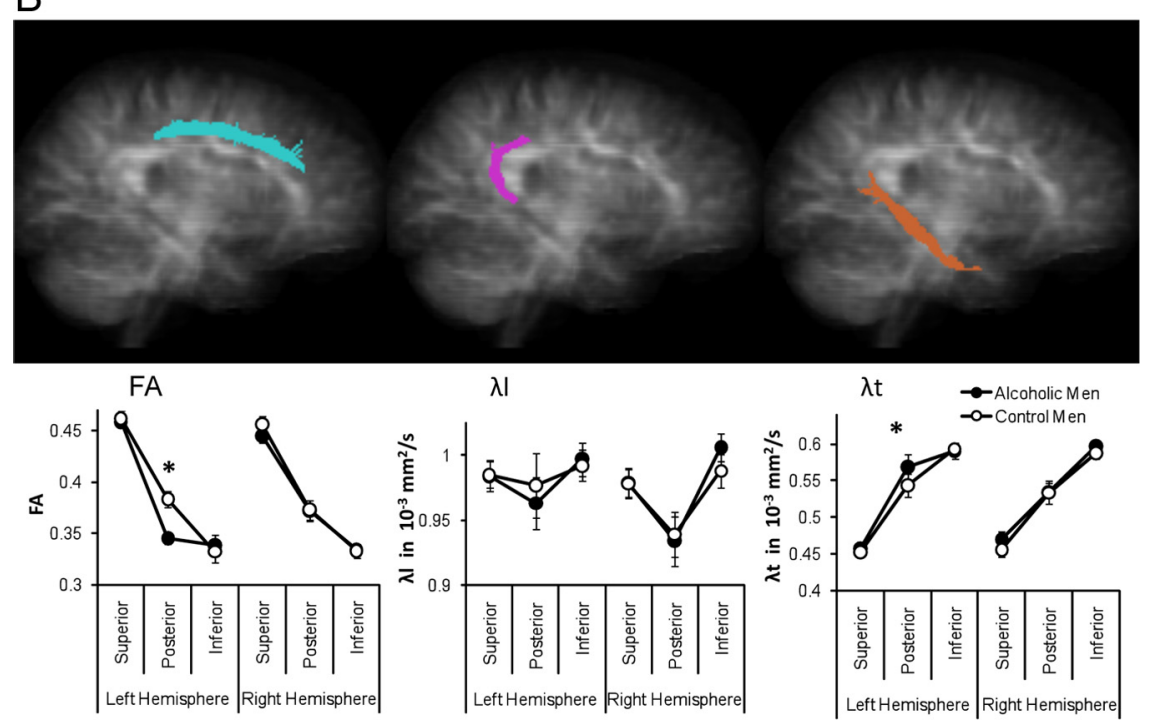

Figure 5. DTI-based visualization of interhemispheric corpus callosum fibers $(\boldsymbol{A})$ divided into seven sectors (prefrontal, frontal, premotor, motor, sensory, parietal, occipital) and intrahemispheric cingulate fibers $(\boldsymbol{B})$ in each cerebral hemisphere divided into three sectors (superior, posterior, and inferior bundles). The mean ( $\pm \mathrm{SE})$ for $F A$, longitudinal $(\lambda \mathrm{I})$, and transverse $(\lambda \mathrm{t})$ diffusivity for each fiber sector and group (control men, alcoholic men) is shown.

group-by-callosal sector ANOVAs for FA, $\lambda \mathrm{l}$, and $\lambda \mathrm{t}$ with group (alcoholics, controls) as between-subject factor and the seven callosal sectors (prefrontal, frontal, premotor, motor, sensory, parietal, occipital) as within-subject factor. For FA, the group main effect $\left(F_{(1,31)}=5.68 ; p=0.023\right)$ and group-by-callosal sector interaction $\left(F_{(1,31)}=4.49 ; p=0.042\right)$ were significant, indicating that the alcoholic group had lower FA than the control group, particularly in the prefrontal and frontal callosal sectors (follow-up $t$ tests; FA prefrontal: $t_{(31)}=2.72, p=0.011$; FA frontal: $\left.t_{(31)}=2.70, p=0.011\right)$. A significant group difference was also found for $\lambda \mathrm{t}\left(F_{(1,31)}=4.19 ; p=\right.$ $0.049)$ but not for $\lambda \mathrm{l}\left(F_{(1,31)}=1.71 ; p=0.2\right)$. However, a significant group-by-callosal sector interaction for $\lambda \mathrm{l}\left(F_{(1,31)}=6.32 ; p=0.017\right)$ and a trend for $\lambda \mathrm{t}\left(F_{(1,31)}=4.04 ; p=0.053\right)$ indicated higher diffusivity in the alcoholic than control group in frontal $\left(\lambda \mathrm{t} 2: t_{(31)}=2.37, p=0.024\right)$ and parietal $\left(\lambda \mathrm{l} 6: t_{(31)}=2.17, p=0.038 ; \lambda \mathrm{t} 6: t_{(31)}=2.33, p=0.026\right)$ callosal sectors. Thus, the interhemispheric connections most affected in this alcoholic group involved regions of prefrontal, frontal, and parietal cortices (Fig. 5A).

\section{Cingulate fiber bundles}

To test for group differences in the integrity of white matter fibers of the corticosubcortical limbic system with posterior bundles connecting to the visual area of medial parieto-occipital cortices within each cerebral hemisphere, we conducted separate group-by-cingulate sector ANOVAs for FA, $\lambda \mathrm{l}$, and $\lambda \mathrm{t}$ with group (alcoholics, controls) as between-subject factor and the cingulate fiber bundle sectors (superior, posterior, inferior) and hemisphere as within-subject factors. Overall, groups did not differ in FA $\left(F_{(1,31)}=0.94 ; p=\right.$ $0.34), \lambda \mathrm{l}\left(F_{(1,31)}=0.003 ; p=0.95\right)$, or $\lambda \mathrm{t}$ $\left(F_{(1,31)}=0.52 ; p=0.48\right)$. However, we observed a significant three-way interaction involving group, hemisphere, and cingulate sector for FA $\left(F_{(1,31)}=10.96 ; p=\right.$ $0.002)$ and $\lambda \mathrm{t}\left(F_{(1,31)}=4.39 ; p=0.044\right)$ but $\operatorname{not} \lambda \mathrm{l}\left(F_{(1,31)}=0.00 ; p=0.98\right)$. Follow-up analyses indicated that alcoholics had lower FA and higher transverse diffusivity $(\lambda \mathrm{t})$ in the left than the right hemisphere, specifically in posterior cingulate fibers (withingroup region-by-hemisphere interaction; FA: $F_{(1,16)}=20.49, p<0.0001 ; \lambda \mathrm{t}: F_{(1,16)}=$ $27.12, p<0.0001$ ), but not controls (FA: $F_{(1,15)}=0.45, p=0.51 ; \lambda \mathrm{t}: F_{(1,15)}=0.36$, $p=0.56)$. Thus, the intrahemispheric connections most affected in this alcoholic group involved left-hemispheric posterior cingulate fibers (Fig. 5B).

\section{Relationships between behavior and fiber integrity \\ CUD and callosal fiber integrity}

Pearson correlations tested whether a small CUD, a behavioral measure of fast interhemispheric transfer time, was related to regional callosal white matter fiber integrity. In controls smaller CUDs were related to lower diffusivity (i.e., greater integrity) in the parietal callosal sector $(\lambda \mathrm{l}: r=0.55, p=0.015)$. Paradoxically, in the alcoholic group, smaller CUDs correlated with greater longitudinal diffusivity (i.e., poorer integrity) in the prefrontal callosal sector $(r=-0.52 ; p=0.017)$ (Fig. 6). To explore this paradox and in view of the larger left- than right-hand CUDs in both groups, we tested for relationships between corpus callosum integrity and CUDs for each hand separately: while smaller left-hand CUDs correlated with higher parietal callosal fiber integrity in the control group (FA: $r=-0.61, p=0.008 ; \lambda \mathrm{t}: r=$ $0.44, p=0.049)$, smaller right-hand CUDs correlated with higher prefrontal $(\lambda \mathrm{l}: r=-0.61, p=0.005 ; \lambda \mathrm{t}: r=-0.46, p=0.031)$ and frontal ( $\lambda \mathrm{l}: r=-0.42, p=0.049)$ callosal diffusivity (i.e., poorer integrity) in the alcoholic group. Thus, in controls, higher posterior callosal fiber integrity was associated with faster left-to-right interhemispheric transfer of information needed by the subdominant right hemisphere to perform the task efficiently (left-hand responses), whereas the dominant left hemisphere (right-hand responses) did not need (right-to-left) callosal input from the subdominant hemisphere. In contrast, in 


\section{CUD}

Corpus Callosum - Parietal Fibers
CTL

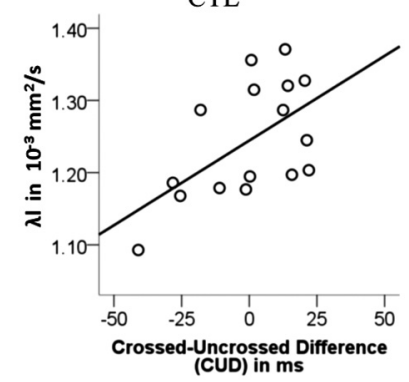

Corpus Callosum - Prefrontal Fibers

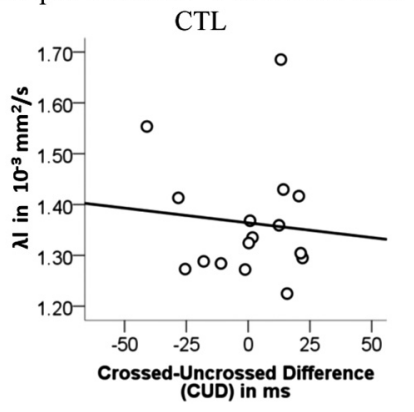

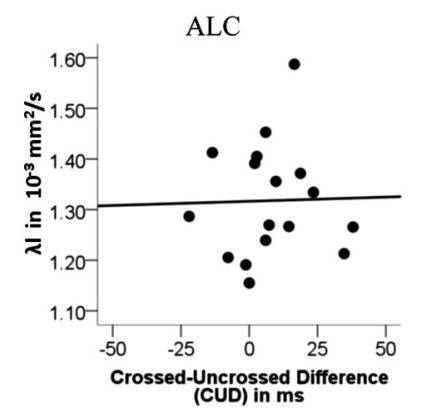

ALC

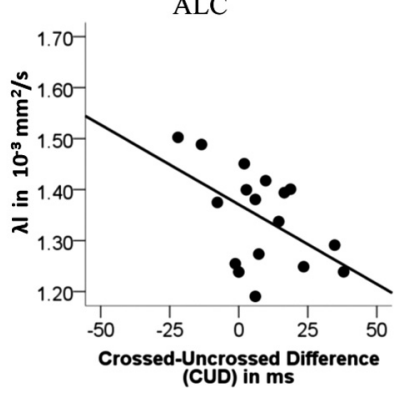

Figure 6. Pearson correlation between the CUD and longitudinal diffusivity of prefrontal and parietal callosal fibers for each group: controls (CTL) and alcoholics (ALC).

alcoholics, greater compromise of anterior callosal fiber integrity was associated with shorter CUD for right-hand responses (i.e., when the dominant left hemisphere was assigned to do the task).

\section{RTE and callosal and cingulate fiber integrity}

Pearson correlations tested whether RTEs, behavioral measures of bilateral and unilateral processing advantages, were related to callosal and cingulate white matter fiber integrity. In the control group, greater bilateral RTEs for left-hand responses correlated with better callosal fiber integrity connecting occipital cortices (FA: $r=0.49, p=0.05 ; \lambda \mathrm{t}: r=-0.53, p=0.035$ ) (Fig. $7 A$ ) (i.e., better posterior callosal fiber integrity was associated with faster summation between hemispheres). Greater unilateral RTEs for the right-hand responses correlated with poorer integrity of lefthemispheric posterior cingulate (RTE: $\lambda \mathrm{l}: r=0.58, p=0.019 ; \lambda \mathrm{t}$ : $r=0.50, p=0.049 ; R(t)$ amplitude: $\lambda \mathrm{l}: r=0.68, p=0.004 ; \lambda \mathrm{t}: r=$ $0.60, p=0.014$ ) (Fig. 7C) and inferior cingulate (RTE: $\lambda \mathrm{t}: r=$ $0.56, p=0.023$ ) (Fig. $7 D$ ) fiber bundles (i.e., better posterior cingulate fiber integrity was associated with less summation within the left hemisphere). In the alcoholic group, in contrast, bilateral processing advantages (large RTEs) correlated with intrahemispheric white matter fiber integrity. In particular, higher left cingulate fiber diffusivity (poorer integrity) correlated with less enhanced RTEs as indicated by lower $R(t)$ amplitudes when responding with the ipsilateral right hand (inferior cingulate; $\lambda \mathrm{l}$ : $r=-0.58, p=0.015)$ and with enhanced RTEs [i.e., higher $R(t)$ amplitudes] when responding with the contralateral left hand (posterior cingulate; $\lambda \mathrm{l}: r=0.61, p=0.009$ ) (Fig. $7 B$ ).

\section{fMRI results}

RTE contrast (pair > single), all conditions

We used fMRI to test whether response time advantage for paired targets compared with single targets (RTE) was reflected by different neural activation for "paired $>$ single" stimulation conditions. Indeed, processing redundant relative to single targets was associated with greater blood oxygenation level-dependent (BOLD) response in bilateral extrastriate cortices in controls. However, alcoholics activated the left extrastriate cortex more to paired than single stimulation (Fig. $8 \mathrm{~A}$ ) and also activated bilateral subcortical gray matter of the thalamus and pallidum and the left cerebellum (supplemental Table 1, available at www.jneurosci.org as supplemental material).

RTE contrasts for bilateral and unilateral stimulation conditions We next tested whether the greater bilateral than unilateral RTEs were also reflected by different neural activation for bilateral and unilateral RTEs (paired > single). For bilateral RTE, controls activated bilateral extrastriate cortices (Fig. $8 \mathrm{~B}$ ), and this activation was higher and more extensive than for unilateral RTE (merged for left and right hemifield stimulation) (Fig. 8C). Controls also activated bilateral thalamic areas for bilateral but not unilateral RTE.

Alcoholics and controls engaged similar regions (see conjunction analyses) (Fig. 8), yet extrastriate cortex activation was smaller and more asymmetrical in alcoholics than controls. Similar to controls, alcoholics engaged bilateral thalamic nuclei for bilateral but not unilateral RTE. A significant interaction between group and RTE laterality (bilateral vs unilateral) indicated that controls specifically engaged left striate, extrastriate, and posterior cingulate areas more than alcoholics for bilateral contrasted with unilateral RTE (Fig. 8D; supplemental Table 1, available at www.jneurosci.org as supplemental material). We next tested the relationship between individual activation differences (bilateral minus unilateral RTE) in left striate, extrastriate, and posterior cingulate areas and the integrity of left posterior cingulate fiber bundles. In controls, greater activation differences (i.e., more activation for bilateral than unilateral RTE) correlated with posterior cingulate fiber integrity, whereas in alcoholics, smaller activation differences (i.e., more activation for unilateral than bilateral RTE) correlated with posterior cingulate fiber integrity (Fig. 8).

\section{RTE contrasts left- and right-hand responses}

In both groups, extrastriate cortex activation during RTE processing was modulated by response hand, with more rightlateralized activation for left-hand responses and more leftlateralized activation for right-hand responses (supplemental Fig. 1, available at www.jneurosci.org as supplemental material). In alcoholics, RTE-related cortical activation was less than in controls. However, for RTE processing with the left (nondominant) hand, alcoholics recruited additional brain areas such as bilateral thalamic nuclei and the cerebellum more than controls. A significant interaction between group and RTE response hand (left vs right) showed that controls engaged the left putamen and temporal cortex regions more than alcoholics when the RTE task was assigned to the left (nondominant) in contrast to the right (dominant) hand (supplemental Table 1, available at www. jneurosci.org as supplemental material).

\section{Summary of the main results}

(1) Behaviorally, chronic alcoholics and controls did not differ in CUD but did so in RTE. Controls showed an asymmetrical response pattern with higher bilateral than unilateral RTE, especially for left-hand responses. This asymmetry was attenuated in alcoholics and shifted toward bilateral over unilateral processing advantages for right- but not left-hand responses. (2) DTI revealed that alcoholics, compared with controls, showed subtle but significant compromise in the integrity of interhemispheric white matter fiber tracts connecting prefrontal, frontal, and pa- 


\section{Bilateral RTE}

A Corpus Callosum - Occipital Fibers CTL

ALC
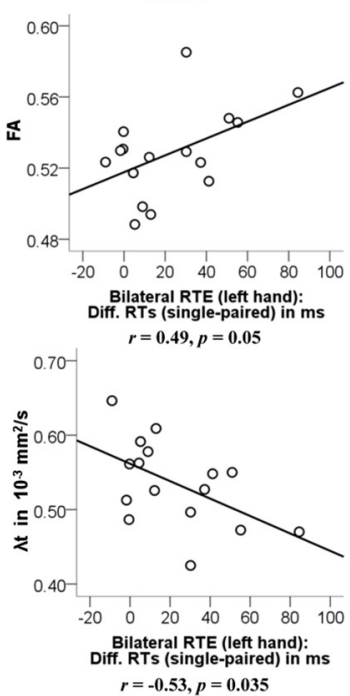

Unilateral RTE

C Left Posterior Cingulate Fibers CTL

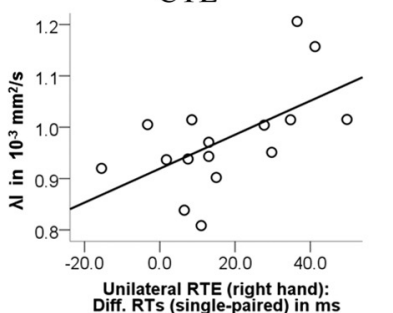

$r=0.58, p=0.019$
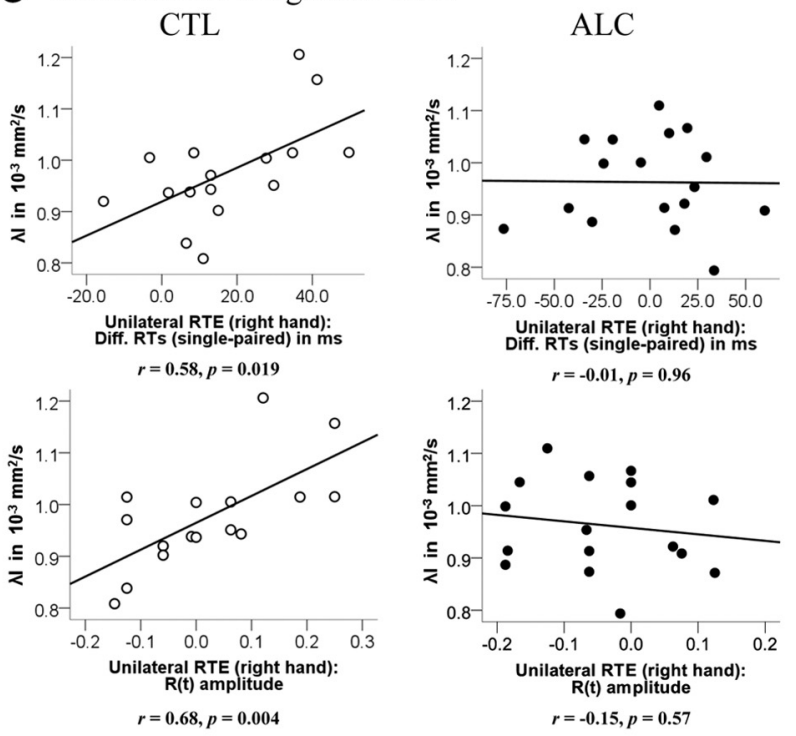

D Left Inferior Cingulate Fibers

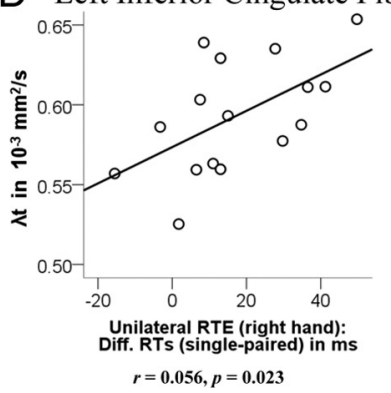

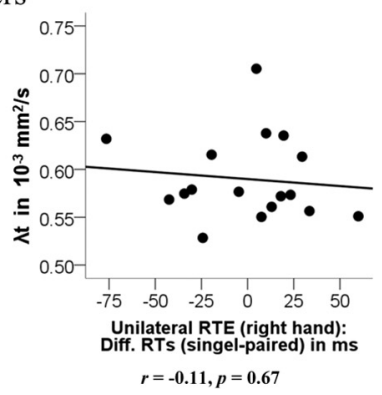

Figure 7. Pearson correlation between the RTEs for bilateral $(\boldsymbol{A}, \boldsymbol{B})$ and unilateral $(\boldsymbol{C}, \boldsymbol{D})$ stimulation and $\mathrm{FA}$ ( $\lambda$ l and $\lambda \mathrm{t}$, longitudinal and transverse diffusivity) of callosal and cingulate fiber sectors for each group: controls (CTL) and alcoholics (ALC).

rietal cortices of each cerebral hemisphere and additional compromise in left-intrahemispheric posterior cingulate fiber tracts connecting the visual areas of medial parieto-occipital cortices with frontal supplementary motor area. (3) Shorter CUDs (i.e., faster IHTT) correlated with greater parietal callosal fiber integrity in controls and with lower prefrontal callosal fiber integrity in alcoholics. (4) In healthy controls, but not in alcoholics, (a) greater bilateral processing advantage of the left hand correlated with higher integrity of fibers crossing the occipital callosal section, and (b) lower unilateral processing advantage for the right hand correlated with lower integrity of the left posterior and inferior cingulate. (5) fMRI revealed abnormally low extrastriate but preserved thalamic activation to RTE in alcoholics relative to controls. (6) Higher left-hemispheric extrastriate/posterior cingulate activation to bilateral over unilateral RTE correlated with higher left posterior cingulate integrity in controls but with lower left posterior cingulate integrity in alcoholics.

\section{Discussion}

Using DTI tractography in combination with fMRI, we have identified an anatomical basis for the modification of normal functional asymmetry in integration of visuomotor information. Even subtle disruption of the integrity of interhemispheric callosal and intrahemispheric cingulate fiber bundles altered hemispheric asymmetry patterns. Specifically, RT speed gained by the bilateral presence of redundant targets was less in alcoholics than controls, especially when responding with the left hand. This processing asymmetry could not be attributed to impaired interhemispheric transfer time as estimated by the CUD, because the groups did not differ on this measure.

Since the fundamental work of Sperry (1961), we have known that visual information cannot transfer from one hemisphere to the other in patients with surgically disconnected cerebral hemispheres, but that performance is preserved, even with commissurotomy, when the required functions can be processed within the hemisphere specialized for the task. Here, we were able to show that visuomotor integration is not an all-or-none process but rather a continuous process that involves both interhemispheric cooperation via posterior corpus callosum fibers and intrahemispheric inhibition via cingulate fibers.

DTI results indicated a regional pattern of white matter fiber compromise and sparing in chronic alcoholism (cf., Pfefferbaum et al., 2009, 2010). For interhemispheric fiber connections, we found that alcoholics showed greatest degradation in callosal fibers linking prefrontal, frontal, and parietal cortices, and for intrahemispheric connections, alcoholics showed greatest degradation in posterior cingulate fibers of the left hemisphere. Posterior cingulate fibers connect limbic circuitry (Morris et al., 1999; Maddock et al., 2003; McCoy et al., 2003), thalamus, cingulate and association cortices, and the hippocampus (Duvernoy, 2005) and also have connections to dorsal and ventral visual pathways 
(Ungerleider and Mishkin, 1982; Vogt et al., 2006). Consistent with the finding of degraded left posterior cingulate fibers in alcoholics, fMRI results showed lower left posterior cingulate and extrastriate cortex activation in alcoholics than controls when comparing BOLD responses to "bilateral" versus "unilateral" redundant targets conditions.

In healthy adults, we demonstrated specific structure-function relationships involving both interhemispheric and intrahemispheric processes: behavioral bilateral processing advantage for the left hand was associated with higher callosal fiber integrity connecting occipital cortices, and behavioral unilateral processing advantage for the right hand was associated with lower integrity of lefthemispheric posterior and inferior cingulate fibers. The examination of fMRI activation patterns demonstrated bilateral extrastriate activations when processing bilateral redundant targets (in contrast to single targets). This activation was less when processing unilateral redundant targets, which corresponds well to less behavioral redundancy gain for unilateral than bilateral paired stimulation. Extrastriate activations were more leftlateralized for right-hand responses and more right-lateralized for left-hand responses, implying that the hemisphere assigned to perform the motor response triggered functional hemispheric asymmetry in visuomotor integration.

Little is known about the functional role of white matter integrity on visuomotor functions. Both positive and negative correlations between motor function and indices of white matter integrity (e.g., FA) in the cingulum (Walther et al., 2010) and corpus callosum (Schulte et al., 2005; Wahl et al., 2007) have been observed, with negative relationships being interpreted as a result of inhibitory mechanisms on motor cortices transmitted by these fibers.

Our finding of behavioral advantages from bilateral but not unilateral RTEs suggests that reciprocal interactions between hemispheres via posterior callosal fibers are beneficial when responding with the nondominant hand. Here, hemispheric resource sharing is required to make this redundant targets process more efficient (Maertens and Pollmann, 2005). In contrast, there is no need for callosal transfer when the task is assigned to the left hemisphere (right-hand responses) that can process the stimuli on its own (Miller, 1982) because of its dominance in finger movements in right-handed individuals (Grafton et al., 2002; Hayashi et al., 2008). These results provide in vivo evidence that interhemispheric and intrahemispheric white matter fiber pathways mediate response facilitation from redundant targets differentially between and within hemispheres in healthy subjects.

The relatively subtle degradation of local white matter fiber microstructure in the alcoholics was adequate to show a relation with behavioral measures of interhemispheric information transfer that indicated abnormal functional asymmetry. Specifically, the normal behavioral asymmetry was attenuated and shifted toward bilateral over unilateral processing advantages for right- but not left-hand responses. This shift in behavioral asymmetry was associated with a shift in structure-function relationships: intrahemispheric and not interhemispheric fiber systems were associated with bilateral processing advantages in alcoholics, i.e., reduced integrity of lefthemispheric posterior and inferior cingulate fibers was correlated with reduced behavioral left-hemispheric (right-hand) and enhanced right-hemispheric (left-hand) processing advantages from bilateral redundant targets. In addition, alcoholics activated the left putamen less than controls when processing RTE and using the left hand compared with the right hand. Because the putamen has been associated with motor preparation (Alexander and Crutcher, 1990; Maillard et al., 2000), lower left putamen activation in alcoholics may have contributed to less left-hemispheric processing advantage.

Consistent with the low behavioral redundancy gain, functional imaging results demonstrated that alcoholics engaged extrastriate cortices less than controls. The combined fMRI-DTI analysis additionally revealed that posterior cingulate fiber integrity predicted the normal pattern of greater bilateral than unilateral activation for visuomotor integration in controls, whereas fiber degradation in alcoholism predicted greater activation for unilateral than bilateral stimulation. The degradation of posterior cingulate fiber integrity may change connectivity to cortical 
sites involved in bottom-up processes for fast response execution to relevant stimuli (Aralasmak et al., 2006). For example, in other functional imaging studies, the posterior cingulate cortex has been the site of higher arousal in alcoholics when processing alcoholrelated stimuli (Tapert et al., 2003) and in cocaine-dependent patients when processing drug-related cues (Kosten et al., 2006).

In a recent DTI study, Putnam et al. (2010) observed in healthy individuals greater right-to-left than left-to-right connectivity between splenium and extrastriate visual cortex, a region implicated in bilateral redundancy gain (Schulte et al., 2006). The observation of asymmetric splenium connectivity is consistent with our finding that occipital callosal fiber connectivity mediated bilateral response facilitation for left-hand responses (right hemisphere) but not for right-hand responses (left hemisphere). We can speculate that the compromise of cerebral white matter in chronic alcoholics (de la Monte, 1988) attenuated this normal hemispheric asymmetry and promoted intrahemispheric processes.

Both of our study groups showed longer RTs to CUDs when responding with the nondominant hand, a finding consistent with other studies showing lower CUDs for right-to-left than left-to-right hemispheric transfer of information (Filbey and Gazzaniga, 1969; Marzi et al., 1991; Braun, 1992). Furthermore, our observation in healthy controls that parietal callosal connectivity was related to CUDs particularly for left-hand responses (when information was transferred from the left to the right hemisphere) provides in vivo support for the hypothesis (Saron and Davidson, 1989; Putnam et al., 2010) that posterior callosal connectivity forms the anatomical basis for hemispheric asymmetry in simple visuomotor interhemispheric transfer time (Saron et al., 2003; Barnett and Corballis, 2005).

These associations seen in controls were not found in alcoholics. Instead, in alcoholics, prefrontal and frontal callosal degradation predicted faster right-to-left hemispheric transfer (shorter CUDs), perhaps reflecting a reorganization of visuomotor processes between and within hemispheres promoted by the subtle but significant degradation of prefrontal, frontal, and parietal callosal connectivity. Thus, callosal white matter degradation, specifically of anterior fibers, appeared to have contributed to altered hemispheric processing preferences in chronic alcoholism.

In summary, by combining microstructural information about white matter fibers with information on neural activation, we demonstrated that visuomotor integration function is related to integrity of interhemispheric and intrahemispheric pathways. This observation is consistent with the idea that functional dynamics of visual cortical areas are constrained by the anatomical characteristics of the underlying white matter tracts (Toosy et al., 2004). Our findings provide clear evidence that white matter fiber pathways between and within hemispheres mediate visuomotor integration asymmetrically and that subtle white matter fiber degradation attenuated the normal pattern of hemispheric asymmetry in chronic alcoholics. These brain structural and functional departures from normality are common concomitants of aging and selective neuropsychiatric conditions (for review, see Sullivan and Pfefferbaum, 2010) and may have ramifications for the efficiency of visual information processing and fast response execution.

\section{References}

Alexander GE, Crutcher MD (1990) Preparation for movement: neural representations of intended direction in three motor areas of the monkey. J Neurophysiol 64:133-150.

American Psychiatric Association (2000) Diagnostic and statistical manual of mental disorders, Ed 4, text revision. Washington, DC: American Psychiatric Association.

Aralasmak A, Ulmer JL, Kocak M, Salvan CV, Hillis AE, Yousem DM (2006) Association, commissural, and projection pathways and their functional deficit reported in literature. J Comput Assist Tomogr 30:695-715.

Barnett KJ, Corballis MC (2005) Speeded right-to-left information transfer: the result of speeded transmission in right-hemisphere axons? Neurosci Lett 380:88-92.

Barrick TR, Lawes IN, Mackay CE, Clark CA (2007) White matter pathway asymmetry underlies functional lateralization. Cereb Cortex 17:591-598.

Basser PJ, Jones DK (2002) Diffusion-tensor MRI: theory, experimental design and data analysis - a technical review. NMR Biomed 15:456-467.

Basser PJ, Pierpaoli C (1998) A simplified method to measure the diffusion tensor from seven MR images. Magn Reson Med 39:928-934.

Bodammer N, Kaufmann J, Kanowski M, Tempelmann C (2004) Eddy current correction in diffusion-weighted imaging using pairs of images acquired with opposite diffusion gradient polarity. Magn Reson Med 51:188-193.

Braun CM (1992) Estimation of interhemispheric dynamics from simple unimanual reaction time to extrafoveal stimuli. Neuropsychol Rev 3:321-365.

Corballis MC, Hamm JP, Barnett KJ, Corballis PM (2002) Paradoxical interhemispheric summation in the split brain. J Cogn Neurosci 14:11511157.

Crovitz HF, Zener K (1962) A group test for assessing hand and eye dominance. Am J Psychol 75:271-276.

de Lacoste MC, Kirkpatrick JB, Ross ED (1985) Topography of the human corpus callosum. J Neuropathol Exp Neurol 44:578-591.

de la Monte SM (1988) Disproportionate atrophy of cerebral white matter in chronic alcoholics. Arch Neurol 45:990-992.

Duvernoy HM (2005) The human hippocampus: functional anatomy, vascularization and serial sections with MRI, Ed 3, pp 26-37. New York: Springer.

Filbey RA, Gazzaniga MS (1969) Splitting the normal brain with reaction time. Psychonom Sci 17:335-337.

Friston KJ, Holmes AP, Poline JB, Grasby PJ, Williams SC, Frackowiak RS, Turner R (1995) Analysis of fMRI time-series revisited. Neuroimage 2:45-53.

Galletti C, Kutz DF, Gamberini M, Breveglieri R, Fattori P (2003) Role of the medial parieto-occipital cortex in the control of reaching and grasping movements. Exp Brain Res 153:158-170.

Gamberini M, Passarelli L, Fattori P, Zucchelli M, Bakola S, Luppino G, Galletti C (2009) Cortical connections of the visuomotor parietooccipital area V6Ad of the macaque monkey. J Comp Neurol 513:622-642.

Gerig G, Corouge I, Vachet C, Krishnan KR, MacFall JR (2005) Quantitative analysis of diffusion properties of white matter fiber tracts: a validation study. Paper presented at 13th Scientific Meeting and Exhibition of the International Society for Magnetic Resonance in Medicine, Miami, FL, May.

Grafton ST, Hazeltine E, Ivry RB (2002) Motor sequence learning with the nondominant left hand. A PET functional imaging study. Exp Brain Res 146:369-378.

Guye M, Parker GJ, Symms M, Boulby P, Wheeler-Kingshott CA, SalekHaddadi A, Barker GJ, Duncan JS (2003) Combined functional MRI and tractography to demonstrate the connectivity of the human primary motor cortex in vivo. Neuroimage 19:1349-1360.

Haaxma R, Kuypers HG (1975) Intrahemispheric cortical connexions and visual guidance of hand and finger movements in the rhesus monkey Brain 98:239-260.

Hayashi MJ, Saito DN, Aramaki Y, Asai T, Fujibayashi Y, Sadato N (2008) Hemispheric asymmetry of frequency-dependent suppression in the ipsilateral primary motor cortex during finger movement: a functional magnetic resonance imaging study. Cereb Cortex 18:2932-2940.

Hofer S, Frahm J (2006) Topography of the human corpus callosum revisited-comprehensive fiber tractography using diffusion tensor magnetic resonance imaging. Neuroimage 32:989-994.

Jenkinson M (2001) Improved unwarping of EPI volumes using regularised $\mathrm{B}_{0}$ maps. Paper presented at Seventh International Conference on Functional Mapping of the Human Brain, Brighton, UK, June.

Jenkinson M (2003) A fast, automated, N-dimensional phase unwrapping algorithm. J Magn Reson Med 49:193-197. 
Kim DH, Adalsteinsson E, Glover GH, Spielman DM (2002) Regularized higher-order in vivo shimming. Magn Reson Med 48:715-722.

Kosten TR, Scanley BE, Tucker KA, Oliveto A, Prince C, Sinha R, Potenza MN, Skudlarski P, Wexler BE (2006) Cue-induced brain activity changes and relapse in cocaine-dependent patients. Neuropsychopharmacology 31:644-650.

Maddock RJ, Garrett AS, Buonocore MH (2003) Posterior cingulate cortex activation by emotional words: fMRI evidence from a valence decision task. Hum Brain Mapp 18:30-41.

Maertens M, Pollmann S (2005) Interhemispheric resource sharing: decreasing benefits with increasing processing efficiency. Brain Cogn 58:183-192.

Maillard L, Ishii K, Bushara K, Waldvogel D, Schulman AE, Hallett M (2000) Mapping the basal ganglia: $\mathrm{fMRI}$ evidence for somatotopic representation of face, hand, and foot. Neurology 55:377-383.

Marzi CA, Bisiacchi P, Nicoletti R (1991) Is interhemispheric transfer of visuomotor information asymmetric? Evidence from a meta-analysis. Neuropsychologia 29:1163-1177.

McCoy AN, Crowley JC, Haghighian G, Dean HL, Platt ML (2003) Saccade reward signals in posterior cingulate cortex. Neuron 40:1031-1040.

Mevorach C, Humphreys GW, Shalev L (2005) Attending to local form while ignoring global aspects depends on handedness: evidence from TMS. Nat Neurosci 8:276-277.

Miller J (1982) Divided attention: evidence for coactivation with redundant signals. Cognit Psychol 14:247-279.

Miller JO (1986) Time course of coactivation in bimodal divided attention tasks. Percept Psychophys 40:331-343.

Mordkoff JT, Yantis S (1991) An interactive race model of divided attention. J Exp Psychol Hum Percept Perform 17:520-538.

Mori S, van Zijl PC (2002) Fiber tracking: principles and strategies-a technical review. NMR Biomed 15:468-480.

Morris R, Petrides M, Pandya DN (1999) Architecture and connections of retrosplenial area 30 in the rhesus monkey (Macaca mulatta). Eur J Neurosci 11:2506-2518.

Neeman M, Freyer JP, Sillerud LO (1991) A simple method for obtaining cross-term-free images for diffusion anisotropy studies in NMR microimaging. Magn Reson Med 21:138-143.

Pandya DN, Seltzer B (1986) The topography of commissural fibers. In: Two hemispheres- one brain: functions of the corpus callosum (Lepore F, Ptito M, Jasper HH, eds), pp 47-73. New York: Wiley.

Park HJ, Kim JJ, Lee SK, Seok JH, Chun J, Kim DI, Lee JD (2008) Corpus callosal connection mapping using cortical gray matter parcellation and DT-MRI. Hum Brain Mapp 29:503-516.

Pfefferbaum A, Rosenbloom M, Crusan K, Jernigan TL (1988) Brain CT changes in alcoholics: effects of age and alcohol consumption. Alcohol Clin Exp Res 12:81-87.

Pfefferbaum A, Rosenbloom M, Rohlfing T, Sullivan EV (2009) Degradation of association and projection white matter systems in alcoholism detected with quantitative fiber tracking. Biol Psychiatry 65:680-690.

Pfefferbaum A, Rosenbloom MJ, Fama R, Sassoon SA, Sullivan EV (2010) Transcallosal white matter degradation detected with quantitative fiber tracking in alcoholic men and women: selective relations to dissociable functions. Alcohol Clin Exp Res 34:1201-1211.

Pierpaoli C, Basser PJ (1996) Toward a quantitative assessment of diffusion anisotropy. Magn Reson Med 36:893-906.

Poffenberger AT (1912) Reaction time to retinal stimulation with special reference to time lost in conduction through nerves center. Arch Psychol 23:1-173.

Poline JB, Worsley KJ, Evans AC, Friston KJ (1997) Combining spatial extent and peak intensity to test for activations in functional imaging. Neuroimage 5:83-96.

Putnam MC, Steven MS, Doron KW, Riggall AC, Gazzaniga MS (2010) Cortical projection topography of the human splenium: hemispheric asymmetry and individual differences. J Cogn Neurosci 22:1662-1669.

Rohlfing T, Maurer CR Jr (2003) Nonrigid image registration in sharedmemory multiprocessor environments with application to brains, breasts, and bees. IEEE Trans Inf Technol Biomed 7:16-25.

Rohlfing T, Zahr NM, Sullivan EV, Pfefferbaum A (2010) The SRI24 multichannel atlas of normal adult human brain structure. Hum Brain Mapp 31:798-819.

Saron CD, Davidson RJ (1989) Visual evoked potential measures of interhemispheric transfer time in humans. Behav Neurosci 103:1115-1138.
Saron CD, Foxe JJ, Simpson GV, Vaughan HG (2003) Interhemispheric visuomotor activation: spatiotemporal electrophysiology related to reaction time. In: The parallel brain: the cognitive neuroscience of the corpus callosum (Zaidel E, Iacoboni M, eds), pp 171-219. Cambridge, MA: MIT.

Schulte T, Sullivan EV, Müller-Oehring EM, Adalsteinsson E, Pfefferbaum A (2005) Corpus callosal microstructural integrity influences interhemispheric processing: a diffusion tensor imaging study. Cereb Cortex 15:1384-1392.

Schulte T, Chen SH, Müller-Oehring EM, Adalsteinsson E, Pfefferbaum A, Sullivan EV (2006) fMRI evidence for individual differences in premotor modulation of extrastriatal visual-perceptual processing of redundant targets. Neuroimage 15:973-982.

Schulte T, Müller-Oehring EM, Javitz H, Pfefferbaum A, Sullivan EV (2008) Callosal compromise differentially affects conflict processing and attentional allocation in alcoholism, HIV, and their comorbidity. Brain Imaging Behav 2:27-38.

Song SK, Sun SW, Ramsbottom MJ, Chang C, Russell J, Cross AH (2002) Dysmyelination revealed through MRI as increased radial (but unchanged axial) diffusion of water. Neuroimage 17:1429-1436.

Sperry RW (1961) Cerebral organization and behavior. Science 133: $1749-1757$.

Stark DE, Margulies DS, Shehzad ZE, Reiss P, Kelly AM, Uddin LQ, Gee DG, Roy AK, Banich MT, Castellanos FX, Milham MP (2008) Regional variation in interhemispheric coordination of intrinsic hemodynamic fluctuations. J Neurosci 28:13754-13764.

Sullivan EV, Pfefferbaum A (2005) Neurocircuitry in alcoholism: a substrate of disruption and repair. Psychopharmacology 180:583-594.

Sullivan EV, Pfefferbaum A (2010) Diffusion tensor imaging in aging and age-related neurodegenerative disorders. In: Diffusion MRI theory, methods, and applications, Ed 1 (Jones DK, ed), pp 624-643. Oxford: Oxford UP.

Sun SW, Liang HF, Trinkaus K, Cross AH, Armstrong RC, Song SK (2006) Noninvasive detection of cuprizone induced axonal damage and demyelination in the mouse corpus callosum. Magn Reson Med 55:302-308.

Tapert SF, Cheung EH, Brown GG, Frank LR, Paulus MP, Schweinsburg AD, Meloy MJ, Brown SA (2003) Neural response to alcohol stimuli in adolescents with alcohol use disorder. Arch Gen Psychiatry 60:727-735.

Tommasi L (2009) Mechanisms and functions of brain and behavioural asymmetries. Philos Trans R Soc Lond B Biol Sci 364:855-859.

Toosy AT, Ciccarelli O, Parker GJ, Wheeler-Kingshott CA, Miller DH, Thompson AJ (2004) Characterizing function-structure relationships in the human visual system with functional MRI and diffusion tensor imaging. Neuroimage 21:1452-1463.

Turken A, Whitfield-Gabrieli S, Bammer R, Baldo JV, Dronkers NF, Gabrieli JD (2008) Cognitive processing speed and the structure of white matter pathways: convergent evidence from normal variation and lesion studies. Neuroimage 42:1032-1044.

Ungerleider LG, Mishkin M (1982) Two cortical visual systems. In: Analysis of visual behavior (Ingle MA, Goodale MI, Masfield RJW, eds), pp 549 586. Cambridge, MA: MIT.

Vogt BA, Vogt L, Laureys S (2006) Cytology and functionally correlated circuits of human posterior cingulate areas. Neuroimage 29:452-466.

Wahl M, Lauterbach-Soon B, Hattingen E, Jung P, Singer O, Volz S, Klein JC, Steinmetz H, Ziemann U (2007) Human motor corpus callosum: topography, somatotopy, and link between microstructure and function. J Neurosci, 27:12132-12138.

Walther S, Federspiel A, Horn H, Wiest R, Dierks T, Strik W, Muller TJ (2010) White matter integrity associated with volitional motor activity. Neuroreport, 21:381-385.

Wandell BA, Dumoulin SO, Brewer AA (2007) Visual field maps in human cortex. Neuron 56:366-383.

Wang JJ, Durazzo TC, Gazdzinski S, Yeh PH, Mon A, Meyerhoff DJ (2009) MRSI and DTI: a multimodal approach for improved detection of white matter abnormalities in alcohol and nicotine dependence. NMR Biomed 22:516-522.

Xu D, Mori S, Solaiyappan M, van Zijl PC, Davatzikos C (2002) A framework for callosal fiber distribution analysis. Neuroimage 17:1131-1143.

Xue R, van Zijl PC, Crain BJ, Solaiyappan M, Mori S (1999) In vivo threedimensional reconstruction of rat brain axonal projections by diffusion tensor imaging. Magn Reson Med 42:1123-1127. 